\title{
SOME GENERALIZATIONS AND COMPLEMENTS OF DETERMINANTAL INEQUALITIES
}

\author{
Hassane AbBas and Mohammad M. GHabries
}

Abstract. K. Audenaert in [1] formulated a determinantal inequality arising from diffusion tensor imaging. Very recently M. Lin proved in [6] a complement and proposed a conjecture. In this short note, we generalize his conjecture and we prove it in a wild case, when the matrix is singular. We also present a refinement of the complement found by Lin and finally we present a series of determinantal inequalities followed by a conjecture.

Mathematics subject classification (2010): 15A45, 15A60.

Keywords and phrases: Determinantal inequality, Hermitian matrix, positive definite matrix, log-majorization, eigenvalues.

\section{REFERENCES}

[1] K. M. R. AudenAert, A determinantal inequality for the geometric mean with an application in diffusion tensor, arXiv 1502.06902v2, 2015.

[2] R. Bhatia, Matrix Analysis, Spring-Verlag, New York, 1997.

[3] R. Bhatia, Positive Definite Matrices, Princeton University Press, New Jersey, 2007.

[4] J. C. Bourin, E.-Y. LEE AND M. Lin, Positive matrices partitioned into a small number of Hermitian blocks, Linear Algebra Appl., 483, 2013, 2591-2598.

[5] J. C. BouRIN, E.-Y. LEE, Matrix inequalities from a two variables functional, Internat. J. Math., 27, 9, 2016, $19 \mathrm{pp}$.

[6] M. Lin, On a determinantal inequality arising from diffusion tensor imaging, Commun. Contemp. Math, 19, 1650044, 2017, 6 pp.

[7] F. Zhang, Matrix Theory: Basic Results and Techniques, Springer, New York, 2nd ed., 2011. 